\title{
VOLUME DA CÂMARA PULPAR DE MOLARES SUPERIORES E INCISIVOS INFERIORES EM IMAGENS DE TOMOGRAFIA COMPUTADORIZADA DE FEIXE CÔNICO
}

\author{
VOLUME OF PULPAR CHAMBER OF SUPERIOR MOLARS AND LOWER \\ INCISIVES IN IMAGES OF CONE BEAM COMPUTED TOMOGRAPHY
}

\author{
Gabriella da Mata SOUZA'; Marco Antônio Zaiden LOUREIRO2; Gustavo Silva CHAVES; Daniel de Almeida DECURCIO³; Julio \\ Almeida SILVA; ${ }^{3}$ Patrícia Correia de SIQUEIRA ${ }^{3}$; Carlos ESTRELA \\ 1 - Graduada em odontologia pela Universidade Federal de Goiás (UFG). \\ 2 - Graduado em odontologia pela Universidade Federal de Goiás (UFG). Mestre em clínica odontológica pela Faculdade de Odontologia, \\ Universidade Federal de Goiás (FO/UFG) e Doutorando em clínica odontológica pela FO/UFG. \\ 3 - Professor (a) Adjunto (a) de Endodontia da Faculdade de Odontologia, Universidade Federal de Goiás (FO/UFG). Especialista, Mestre e Doutor \\ em Endodontia. \\ 4 - Professor Titular de Endodontia da Faculdade de Odontologia da Universidade Federal de Goiás (FO/UFG). Especialista, Mestre e Doutor em \\ Endodontia.
}

\section{RESUMO}

Objetivo: Determinar o volume da câmara pulpar de molares superiores e incisivos inferiores humanos extraídos em imagens obtidas de tomografia computadorizada de feixe cônico (TCFC). Material e métodos: Foram selecionados 15 molares superiores e 15 incisivos inferiores humanos. Os dentes foram posicionados em suporte de resina acrílica e submetidos aos exames. Posteriormente, foi realizada a avaliação do volume das câmaras pulpares, em milímetros cúbicos, utilizando o software InVesalius ${ }^{\circledR}$. Para este cálculo, foi empregado um método de ajuste manual, no qual foram aplicados filtros de exclusão das estruturas que não fizessem parte das câmaras pulpares. Os dados obtidos foram tabulados e analisados no programa BioEstat versão 5.3. Utilizou-se o teste t para comparar os volumes das câmaras pulpares entre os espécimes, com um nível de significância de $5 \%$. Resultados: O valor médio de volume encontrado nos incisivos inferiores foi de 1,11 $\mathrm{mm}^{3}$ e nos molares superiores de 28,28 $\mathrm{mm}^{3}$. Observou-se diferença estatisticamente significante entre o volume da câmara pulpar dos incisivos inferiores em comparação a dos molares superiores $(\mathrm{p}<0,05)$. Conclusões: A análise em TCFC associada a software com ferramentas específicas de medida constitui um recurso aplicável para determinação do volume da câmara pulpar. Foram encontrados volumes significativamente maiores em molares superiores quando comparados aos incisivos inferiores.

PALAVRAS-CHAVE: Endodontia; Tomografia computadorizada de feixe cônico; Tratamento do canal radicular.

\section{INTRODUÇÃO}

A avaliação do estado em que se encontra o tecido pulpar é fundamental para o planejamento do tratamento endodôntico. Os testes térmicos ao frio e ao calor, o teste elétrico e o teste de cavidade são os recursos convencionais frequentemente utilizados para avaliar a condição pulpar dos dentes humanos. Todavia, estes recursos semiotécnicos apresentam diversas limitações, como a possibilidade de resultados falsos positivos ou falsos negativos, em dentes expostos a traumatismos dentários, além de gerar sensações desagradáveis aos pacientes, decorrente de estímulos às fibras nervosas induzidas pelo teste frio ou ao calor ${ }^{1}$. Nesse contexto, recursos tecnológicos têm auxiliado o aprimoramento de ferramentas valiosas para o diagnóstico do estado fisiológico pulpar, por meio de espectrofotometria, fluxometria por laser doopler e a oximetria de pulso ${ }^{1-4}$.
A verificação do percentual de saturação de oxigênio do tecido pulpar tem sido determinada a partir de adaptações do oxímetro de pulso utilizado na medicina, acomodado ao dente do indivíduo ${ }^{4-6}$. Porém, novos estudos envolvendo as particularidades da aplicação do oxímetro de pulso diretamente no órgão dental, podem possibilitar um melhor entendimento da relação entre o volume da câmara pulpar e o nível de saturação de oxigênio da polpa. Desta maneira, o conhecimento aprofundado da morfologia interna é fundamental para se entender o comportamento do tecido pulpar?.

O volume da câmara pulpar dos dentes humanos pode ser verificado utilizando ferramentas específicas dos softwares utilizados para a manipulação de exames por imagens em odontologia. A tomografia computadorizada de feixe cônico (TCFC) constitui um recurso preciso para a realização de diagnóstico 
em endodontia ${ }^{8}$, podendo fornecer modelos tridimensionais ${ }^{9}$, para análise de volume com softwares específicos, como o Invesalius ${ }^{\circledR}$ (Centro de Tecnologia da Informação Renato Archer, Campinas, São Paulo). A utilização da TCFC em softwares específicos para a reconstrução de modelos tridimensionais que permitem realizar medidas de volume representa forte justificativa para o presente estudo.

O objetivo deste trabalho foi determinar o volume da câmara pulpar de molares superiores e incisivos inferiores humanos por meio de imagens de tomografia computadorizada de feixe cônico.

\section{MATERIAL E MÉTODOS}

\section{Seleção da amostra}

Foram selecionados 15 molares superiores e 15 incisivos inferiores humanos extraídos para compor a amostra do estudo. Os dentes foram doados por pacientes atendidos no serviço de Urgência da Faculdade de Odontologia da Universidade Federal de Goiás e com indicação de exodontia por motivos diversos. Os dentes foram coletados após a leitura, compreensão e assinatura de um Termo de Consentimento Livre e Esclarecido (TCLE) pelo paciente, resguardando sigilo de identidade, privacidade e confidencialidade.

Os dentes extraídos foram imersos em solução de hipoclorito de sódio 5\% (Fitofarma, Lt. 20442, Goiânia, GO, Brasil) por 30 minutos, para remoção de tecidos orgânicos e, posteriormente, acondicionados em frasco contendo solução de timol 0,2\%. Foram incluídos na amostra primeiros e segundos molares superiores e incisivos centrais e laterais inferiores. Radiografias periapicais digitais foram realizadas (Aparelho Diox e Sensor digital T1 - Microimagem, Santa Catarina, Brasil) para verificação dos critérios de exclusão, que foram: presença de cárie ou restaurações extensas, fraturas, dentes com cálculos pulpares e/ou canais radiculares obliterados, dentes com tratamento endodôntico e reabsorções radiculares.

\section{Aquisição das imagens de TCFC}

Para a aquisição das imagens por TCFC, os dentes foram posicionados em resina acrílica (Jet, Campo Limpo Paulista, SP, Brasil) para melhor estabilização dos dentes, padronização das imagens, além da individualização dos espécimes, permitindo a separação entre os mesmos. As imagens foram obtidas em aparelhos de alta resolução PreXion 3D Inc. (San Mateo, CA, EUA) configurado para aquisição em espessura de $0,100 \mathrm{~mm}$ (dimensões 1,170 mm × 1,570 mm × 1,925 mm), FOV de 56,00 mm, voxel de $0,100 \mathrm{~mm}, 33,5 \mathrm{~s}$. A voltagem foi de $90 \mathrm{kVp}$, a corrente de 4 $\mathrm{mA}$ e o tempo de exposição foi de 33,5 segundos.

\section{Avaliação de volume da câmara pulpar}

As avaliações do volume das câmaras pulpares foram realizadas utilizando o software InVesalius ${ }^{\circledR}$ (Centro de Tecnologia da Informação Renato Archer, Campinas, SP, Brasil) em um computador Acer com processador Intel ${ }^{\circledR}$ Core i3-2348M (2.3 GHz, 3MB L3 cache) e sistema operacional de 32 bits (Windows 8.1 Pro).

Primeiramente, foi realizado um ajuste individual, para que cada dente ficasse com seu longo utilizando ferramentas de filtro para voxels de densidade compatíveis com a dentina, o esmalte, a polpa e o suporte de resina acrílica utilizado para estabilizar os espécimes. No software InVesalius ${ }^{\circledR}$, foram estabelecidos, na configuração thereshold, os valores de densidade radiográfica mínimo e máximo, respectivamente: -900 e 1000, para os molares superiores e -900 e 1500, para os incisivos inferiores. Esse limiar foi estabelecido aumentando-se gradativamente o valor máximo de densidade, até que o esmalte e a dentina fossem totalmente excluídos da imagem. Para a análise do volume das câmaras pulpares, foi empregado um método de ajuste manual, no qual foram aplicados filtros de exclusão das estruturas que não fizessem parte das câmaras pulpares dos dentes. No grupo de molares superiores, utilizou-se como referências para a delimitação da câmara pulpar os cornos pulpares na parede oclusal, as paredes circundantes e o plano correspondente à região de entrada dos canais radiculares (Figura 1A). No grupo de incisivos inferiores, a câmara pulpar foi delimitada pela região mais incisal, paredes circundantes e o plano correspondente à junção amelocementária (Figura 1B). Após a delimitação da câmara pulpar de cada espécime, foi aplicada a ferramenta para o cálculo de volume em milímetros cúbicos.

\section{Análise estatística}

Os dados obtidos foram tabulados e analisados no programa BioEstat versão 5.3. Inicialmente os dados foram testados quanto à normalidade (teste de Kolmogorov-Smirnov). Verificada a distribuição paramétrica, foi utilizado o teste $\mathrm{T}$ para comparar os volumes da câmara pulpar entre incisivos inferiores e molares superiores, adotando um nível de significância de 5\% $(\alpha=0,05)$.

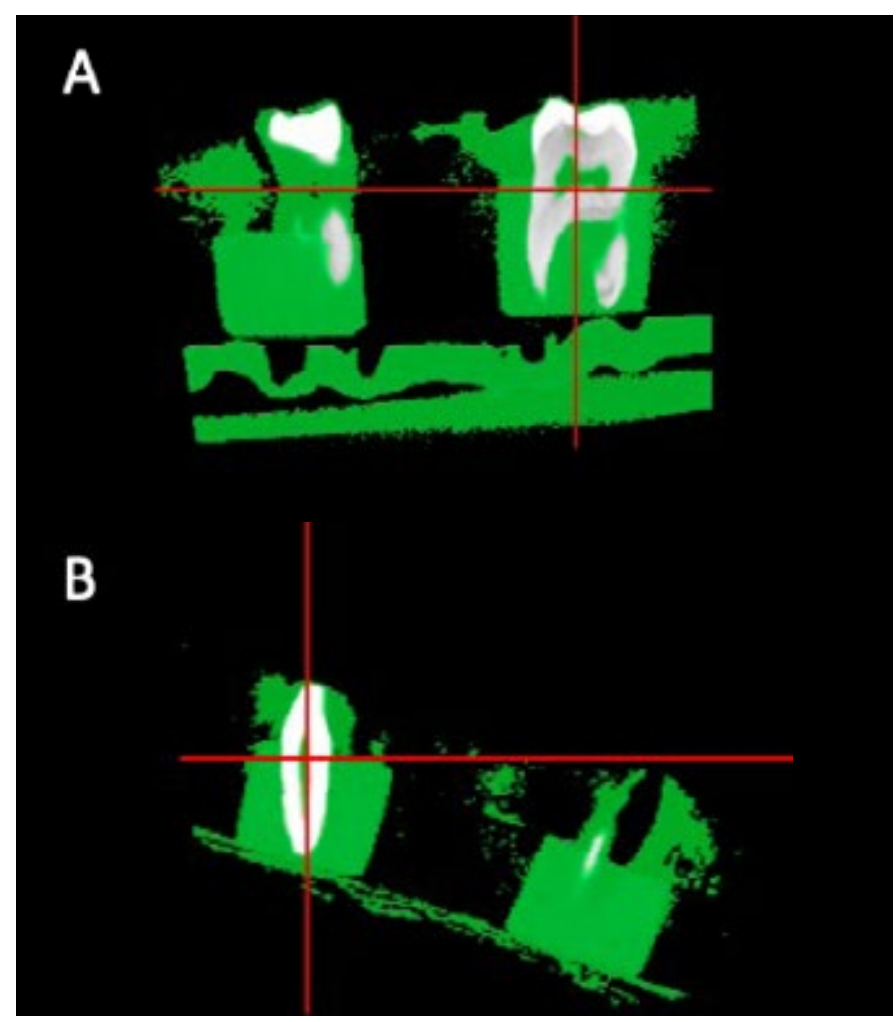

Figura 1 - Determinação do plano que separa a câmara pulpar dos canais radiculares (linhas horizontais em vermelho) nos molares superiores - plano correspondente à região de entrada dos canais radiculares (A) e nos incisivos inferiores - plano correspondente à posição da junção amelocementária (B) 


\section{RESULTADOS}

Uma representação das imagens obtidas no software InVesalius ${ }^{\circledR}$ após a reconstrução tridimensional da câmara pulpar do molar superior e do incisivo inferior está apresentada na Figura 2.

Os valores de média, desvio padrão, mínimo e máximo em milímetros cúbicos, dos dados encontrados estão apresentados na Tabela 1. Observou-se diferença estatisticamente significante entre o volume da câmara pulpar dos incisivos inferiores em comparação a dos molares superiores $(p<0,05)$ (Figura 3).

\section{DISCUSSÃO}

As variações da anatomia entre os diferentes grupos de dentes podem influenciar nos valores de saturação de oxigênio. O volume da câmara pulpar, a espessura de esmalte e dentina são exemplos de fatores que podem interferir na leitura do oxímetro de pulso. Desta maneira, o aprofundamento do conhecimento nas câmaras pulpares de grupos dentários distintos é necessário para determinar a influência desses fatores biofísicos na oximetria ${ }^{10}$.

Com a finalidade de aprofundar o estudo na morfologia interna dos dentes e consequentemente, nas particularidades da aplicação do oxímetro de pulso diretamente no órgão dental, no presente estudo foram realizadas as avaliações das medidas de volume da câmara pulpar de molares superiores e incisivos inferiores humanos, a fim de contribuir com informações cabíveis ao diagnóstico endodôntico, pois constitui importância para o correto planejamento e execução de um procedimento operatório.

A carência de softwares apropriados para a análise de imagens Digital Imaging and Communications in Medicine (DICOM) geradas pelos exames de imagem em TCFC, representava uma limitação para os estudos que envolviam reconstrução tridimensional. Os softwares disponíveis eram onerosos e consequentemente, não acessíveis aos usuários comuns. Entretanto, com o surgimento dos softwares livres, houve a redução de custos e homogeneização do uso multicêntrico, possibilitando o progresso contínuo e promovendo o desenvolvimento de diversas linhas de pesquisa ${ }^{11}$.

O InVesalius ${ }^{\circledR}$ é um software livre, desenvolvido no Brasil pelo Centro de Tecnologia da Informação Renato Archer (CTI), e seu acesso está disponível no Portal do Software Público. O programa tem sido utilizado em diversas áreas da saúde, pois apresenta a capacidade de operar nas plataformas Linux, Windows, e Apple Mac OS X, além de obter imagens 2D, provenientes de equipamentos como tomografia computadorizada e ressonância magnética e gerar modelos 3D correspondentes às estruturas anatômicas, obter volume e segmentação semiautomática e manual ${ }^{11}$.

O software InVesalius ${ }^{\circledR}$ foi o programa selecionado no presente estudo, para a realização das medidas de volume dos dois grupos de dentes. Tolentino et al. ${ }^{12}$ (2018), verificaram que as medições lineares nos softwares XoranCat ${ }^{\circledR}$, Radiant ${ }^{\circledR}$ e InVesalius ${ }^{\circledR}$ mostraram-se confiáveis e precisas, quando comparadas com as medições físicas. O software mais preciso foi o InVesalius ${ }^{\circledR}$, dados que reforçaram a sua utilização no presente estudo.

O conhecimento da anatomia interna dos dentes constitui elemento para o sucesso do tratamento endodôntico. Nesse sentindo, vários estudos se propuseram a analisar a anatomia interna nos dentes permanentes ${ }^{13-16}$. Entretanto, poucos estudos dedicaram-se em analisar o volume da câmara pulpar, dados que permitem dar início à resolução de questões ainda não explicadas na endodontia.

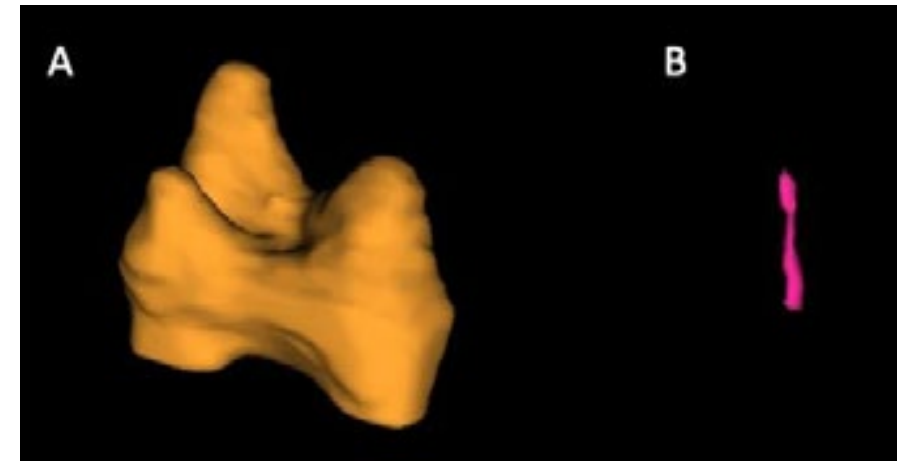

Figura 2 - Imagem obtida no software InVesalius ${ }^{\circledR}$ após a reconstrução tridimensional da câmara pulpar de molar superior (A) e do incisivo inferior (B)

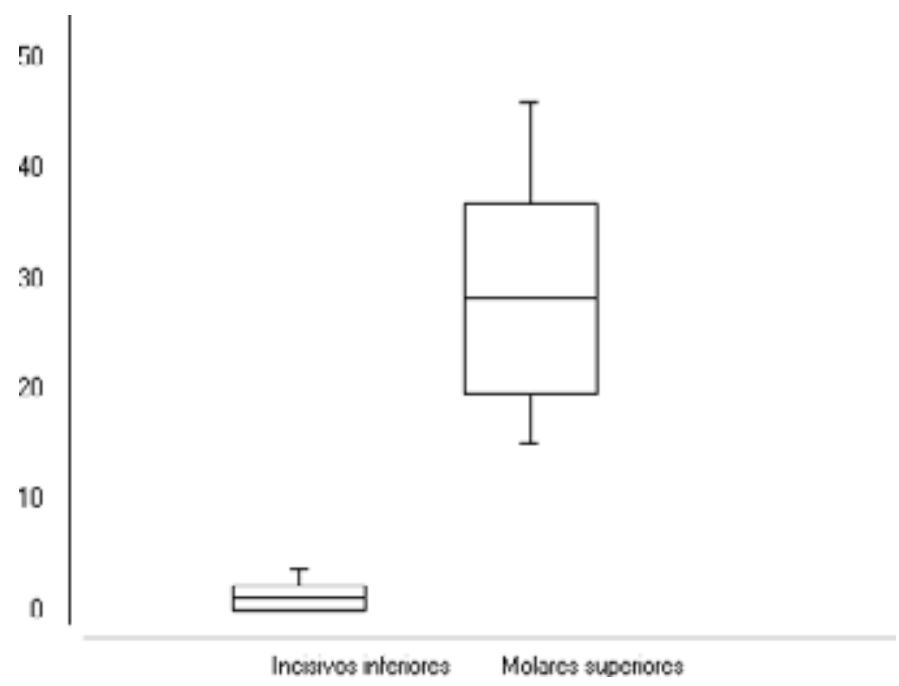

Figura 3 - Gráfico representando a distribuição dos volumes encontrados nos incisivos inferiores e molares superiores

Tabela 1 - Valores de volume em $\mathrm{mm}^{3}$ encontrados nos incisivos inferiores e molares superiores

\begin{tabular}{llllll}
\hline & $\mathrm{n}$ & Média & Desvio-padrão & Mínimo & Máximo \\
\hline Incisivos inferiores & 15 & $1.11 \mathrm{~A}$ & 1.08 & 0.001 & 3.753 \\
\hline Molares superiores & 15 & $28.28 \mathrm{~B}$ & 8.62 & 15.095 & 46.067 \\
\hline
\end{tabular}

Letras diferentes representam diferença estatisticamente significante pelo teste $t(p<0,05)$.

Neste estudo, foi possível analisar 30 elementos dentários, divididos em dois grupos: 15 incisivos inferiores e 15 molares superiores, dos quais foram obtidas informações em $\mathrm{mm}^{3}$ do volume da câmara pulpar. A presente pesquisa mostrou que existe uma diferença significativa entre os volumes dos grupos estudados, resultado este já esperado, visto que a morfologia dos espécimes é diferente. No geral, os incisivos inferiores contêm um contorno trapezoidal alongado, quase retangular e as bordas mesial e distal, tendem a ficar paralelas. Já os molares superiores, apresentam contorno trapezoidal, de base maior voltada para oclusal, os lados mesial e distal convergem a partir do ponto de contato para a cervical ${ }^{17}$.

As avaliações do volume das câmaras pulpares, no presente estudo, foram realizadas apenas por um avaliador, pois trata-se de imagens com dimensões muito pequenas, o que dificulta a 
repetição das medições, além de estabelecer variáveis de confusão (vieses) para delimitar corretamente o ponto de referência. Uma vez que a medição é repetida, dificilmente as referências serão delimitadas na posição correta ${ }^{18}$.

Uma limitação desse estudo consistiu na seleção de elementos dentários de pacientes de ambos os gêneros e com diferentes faixas etárias, visto que a amostra foi de conveniência, de acordo com a necessidade e disponibilidade dos pacientes atendidos no serviço de urgência da Faculdade de Odontologia da Universidade Federal de Goiás. Sabe-se que o volume da cavidade pulpar diminui com o passar dos anos, devido à deposição de dentina secundária e terciária, formada ao longo da vida do indivíduo ${ }^{19-22}$. Entretanto, a informação que o sexo possa influenciar significativamente no tamanho da câmara pulpar, parece ser controvérsia. Estudos de Limdiwala e Shah ${ }^{21}$ (2013) e Karkhanis et al..$^{23}$ (2013) não encontraram diferenças provocadas pelo gênero, resultado contraditório com os de Agematsu et al. ${ }^{24}$ (2010) e Igbigbi e Nyirenda ${ }^{25}$ (2005), que mencionaram a influência do estrogênio na formação da dentina secundária.

A tomografia computadorizada de feixe cônico é considerada um método preciso, prático e não destrutivo ${ }^{26}$, além disso, o seu amplo uso na prática odontológica permite verificar informações in vivo através de reconstruções 3D da dentição humana ${ }^{22}$. Estas características viabilizam novas pesquisas diretamente em pacientes, com o potencial de aprofundar o conhecimento da morfologia dental e suas variáveis e, consequentemente, frente à aplicação do oxímetro de pulso como recurso semiotécnico na análise do estado pulpar.

\section{CONCLUSÕES}

A análise em TCFC associada a software com ferramentas específicas de medidas constitui um recurso aplicável para determinação do volume da câmara pulpar dos dentes. Foram encontrados volumes significativamente maiores em molares superiores quando comparados aos incisivos inferiores.

\section{REFERÊNCIAS}

01. Ciobanu G, Ion I, Ungureanu L. Testing of pulp vitality by pulsoximetry. Int J Med Dent Case Reports. 2012; 16(2): 94-98.

02. Calil E, Caldeira CL, Gavini G, Lemos EM. Determination of pulp vitality in vivo with pulse oximetry. Int Endod J. 2008; 41(9): 741-746.

03. Jafarzadeh $H$, Rosenberg PA. Pulse oximetry: review of potencial aid in endodontics diagnosis. J Endod. 2009; 35(3): 329-333.

04. Estrela C, Serpa GC, de Alencar AHG, Bruno KF, Barletta FB, Felippe WT, et al. Oxygen saturation in the dental pulp of maxillary premolars in different age groups - Part 1. Braz Dent J. 2017; 28(5): 573-577.

05. Gopikrishna V, Tinagupta K, Kandaswamy D. Comparison of electrical, termal, and pulse oximetry methods for assessing pulp vitality in recently traumatized teeth. J Endod. 2007; 33(5): 531-535.

06. Giovanella LB, Barletta FB, Felippe WT, Bruno KF, Alencar AH, Estrela C. Assessment of oxygen saturation in dental pulp of permanente teeth with periodontal disease. J Endod. 2014; 40(12):1927-1931.

07. Mauger MJ, Schinler WG, Walker WA. An evaluation of canal morphology at different levels of root resection in mandibular incisors. J Endod. 1998; 24(9): 607-609.

08. Estrela C, Bueno MR, Leles CR, Azevedo B, Azevedo. Accuracy of Cone Beam Computed Tomography and Panoramic and Periapical
Radiography for detection of Apical Periodontitis. J Endod. 2008; 34(3): 273-279.

09. Garib DG, Raymundo Junior R, Raymundo MV, Raymundo DV, Ferreira SN. Tomografia computadorizada de feixe cônico (Cone beam): entendendo este novo método de diagnóstico por imagem com promissora aplicabilidade na Ortodontia. Rev Dent Press Ortod Ortop Facial. 2007; 12(2): 139-156.

10. Siqueira PC, Decurcio DA, Silva JA, Alencar AHG, Rossi-Fedele G, Estrela C. Oximetria de pulso na avaliação da vitalidade pulpar: análise crítica. Robrac. 2018; 27(81): 61-67.

11. Costa ALF, Yasuda CL, Nahás-Scocate ACR. Utilização de softwares livres para visualização e análise de imagens 3D na Odontologia. Rev Assoc Paul Cir Dent. 2016; 70(2): 151-155.

12. Tolentino ES, Yamashita FC, Albuquerque S, Walewski LA, Iwaki LCV, Takeshita WM, et al. Reliability and accuracy of linear measurements in cone-beam computed tomography using different software programs and voxel sizes. J Conserv Dent. 2018; 21(6): 607-612.

13. Silva EJ, Nejaim Y, Silva AI, Haiter-Neto F, Zaia AA, Cohenca N. Evaluation of root canal configuration of maxillary molars in a brazilian population using cone-beam computed tomographic imaging: an in vivo study. J Endod. 2014; 40(2): 173-176.

14. Marceliano-Alves M, Alves FR, Mendes DM, Provenzano JC. Micro-computed tomography analysis of the root canal morphology of palatal roots of maxillary first molars. J Endod. 2016; 42(2): 280-3.

15. Divine KA, Mcclanahan SB, Fok A. Anatomic analysis of palatal roots of maxillary molars using micro-computed tomography. J Endod. 2019; 45(6): 724-728.

16. Mirhosseini F, Tabrizizadeh M, Nateghi N, Rad ES, Derafshi A, Ahmadi B, et al. Evaluation of root canal anatomy in mandibular incisors using cbct imaging technique in an iranian population. J Dent (Shiraz). 2019; 20(1): 24-29.

17. Madeira MC, Rizzolo RJC. Anatomia do dente. 7. Ed. Anatomia individual dos dentes: descrição anatômica de dentes permanentes. [s.I.]: Sarvier; 2014. p. 35-61. Cap. 2.

18. Bueno MR, Estrela CRA, Granjeiro JM, Sousa-Neto MD, Estrela C. Method to determine the root canal anatomic dimension by using a new cone-beam computed tomography software. Braz Dent J. 2019; 30(1): 3-11.

19. Carvalho TS, Lussi A. Age-related morphological, histological and functional changes in teeth. J Oral Rehabil. 2017; 44(4): 291-298.

20. Gotmare SS, Shah T, Periera T, Waghmare MS, Shetty S, Sonaware $S$, et al. The coronal pulp cavity index: a forensic tool for age determination in adults. Dent Res J. 2019; 16(3): 160-165.

21. Limdiwala PG, Shah JS. Age estimation by using dental radiographs. J Forensic Dent Sci. 2013; 5(2): 118-122.

22.Zhi-Pu G, Pan Y, Gang L, Ji-Zong Z, Xu-Chen M. Age estimation based on pulp cavity/chamber volume of 13 types of tooth from cone beam computed tomography images. Int J Legal Med. 2016; 130(4): 1159-1167.

23. Karkhanis S, Mack P, Franklin D. Age estimation standards for a Western Australian population using the coronal pulp cavity index. Forensic Sci Int. 2013; 231(1-3): 412-416.

24. Agematsu $\mathrm{H}$, Someda $\mathrm{H}$, Hashimoto $M$, Matsunaga S, Abe S, Kim HJ, et al. Three- dimensional observation of decrease in pulp cavity volume using micro-CT: age-related change. Bull Tokyo Dent Coll. 2010; 51(1): 1-6. 
25. Igbigbi PS, Nyirenda SK. Age estimation of Malawian adults from dental radiographs. West Afr J Med. 2005; 24(4): 329-333.
26. Cotton TP, Geisler TM, Holden DT, Schwartz SA, Schindler WG. Endodontic applications of cone-beam volumetric tomography. J Endod. 2007; 33(9): 1121-1132.

\section{ABSTRACT}

Objective: To determine the pulp chamber volume of human upper and lower incisor molars extracted from cone beam computed tomography (CBCT) images. Material and methods: Fifteen maxillary molars and 15 human lower incisors were selected. The teeth were positioned in acrylic resin support and submitted to imaging exams. Subsequently, the pulp chamber volume was evaluated in cubic millimeters using InVesalius ${ }^{\circledR}$ software. For this calculation, a manual adjustment method was employed, in which filters were applied to exclude structures that were not part of the pulp chambers. The data obtained were tabulated and analyzed using the BioEstat version 5.3 software. The t-test was used to compare pulp chamber volumes between specimens, with a significance level of $5 \%$. Results: The mean volume value found in the lower incisors was $1.11 \mathrm{~mm}^{3}$ and in the upper molars $28.28 \mathrm{~mm}^{3}$. There was a statistically significant difference between the pulp chamber volume of the lower incisors compared to the upper molars $(\mathrm{p}<0.05)$. Conclusions: The CBCT analysis associated with software with specific measurement tools is an applicable resource for pulp chamber volume determination. Significantly higher volumes were found in maxillary molars when compared to mandibular incisors.

KEYWORDS: Endodontics; Cone beam computed tomography; Root canal treatment.

\section{AUTOR PARA CORRESPONDÊNCIA}

Patrícia Correia de Siqueira

Faculdade de Odontologia (FO/UFG)

Avenida Universitária esquina com $1^{a}$ Avenida, $\mathrm{s} / \mathrm{n}^{\circ}$, Setor

Leste Universitário, Campus Colemar e Silva, Goiânia-GO,

CEP: 74605-220

E-mail: patricia.csiqueira@hotmail.com 